Proceedings

\title{
Development of a Printed E-Textile for the Measurement of Muscle Activation via EMG for the Purpose of Gesture Control ${ }^{+}$
}

\author{
David Court and Russel Torah *
}

Citation: Court, D.; Torah, R. Development of a Printed E-Textile for the Measurement of Muscle Activation via EMG for the Purpose of Gesture Control. Proceedings 2021, 68, 8. https://doi.org/10.3390/ proceedings 2021068008

Published: 13 January 2021

Publisher's Note: MDPI stays neutral with regard to jurisdictional claims in published maps and institutional affiliations.

Copyright: $₫ 2021$ by the authors. Licensee MDPI, Basel, Switzerland. This article is an open access article distributed under the terms and conditions of the Creative Commons Attribution (CC BY) license (http://creativecommons.org/licenses /by/4.0/).

\author{
Electronics and Computer Science, University of Southampton, Southampton SO17 1BJ, UK; \\ dwc1g17@soton.ac.uk \\ * Correspondence: rnt@ecs.soton.ac.uk \\ + Presented at the International Conference on the Challenges, Opportunities, Innovations and Applications \\ in Electronic Textiles (E-Textiles 2020), Virtual venue, UK, 4 November 2020.
}

\begin{abstract}
This paper details the development of an e-textile gesture controller using screen-printed electrodes to measure Electromyography (EMG); the electrical signals produced in a muscle during its use. The final e-textile consists of 7 fabric electrodes able to take measurements from three muscular groups in the right forearm. When accompanied with processing circuitry, also produced in this study, a total of five gestures are uniquely identified with an average accuracy of $\sim 93 \%$ when operating with a switching delay of $150 \mathrm{~ms}$ or greater.
\end{abstract}

Keywords: electromyography; gesture control; screen-printing; e-textiles; wearable healthcare

\section{Introduction}

Electromyography (EMG) is the measurement of electrical activity in the muscles. Traditionally, EMG readings are taken in a clinical setting using single-use stick-on electrodes (usually a pair of electrodes per muscle) or via dry-contact rigid sensor modules such as the commercial MYO armband gesture controller [1]. This study incorporates screen-printed fabric electrodes allowing continual reuse whilst being flexible and breathable, providing comfort to the user. This study shows the e-textile technology controlling a computer mouse, demonstrating how it could be used in a wide range of consumer electronics or prosthesis.

\section{E-Textile EMG Measurement System Design}

The full EMG measurement system is outlined in Figure 1. Each electrode pair output is processed by an instrumentation amplifier with additional inverting amplifier stage. A coupling capacitor is included at the input of each inverting amplifier to remove the constant DC value present between the two electrodes forming one pair. Only once this DC component has been removed from each of the pairs can the signals be multiplexed allowing for all three to share common stages of the processing circuitry. When connecting the multiplexing stage directly from the sensors the variations of DC voltages gave a significant settling time when switching, hence the DC offset must be removed and the initial stages of the processing circuitry must be duplicated.

Following this, two filtering stages are incorporated; the first is a $50 \mathrm{~Hz}$ notch filter to remove mains noise. The second is a $20-500 \mathrm{~Hz}$ bandpass filter. The lower cut-off removes low frequency interference which is likely to be caused by the movement of the electrodes on the skin (motion artefacts), while the higher cut-off removes high frequency noise from the surrounding environment.

Following the filtering stages, the signal enters an envelope detector which extracts the amplitude from the "raw" EMG signal, thus measuring the level of activation in the 
muscle. Once the amplitude is extracted it is passed via a non-inverting amplifier before being read by the microcontroller.

The microcontroller reads in the analogue voltage and assesses whether the muscle being monitored is classed as active or inactive, based on predetermined thresholds. The microcontroller also selects which electrode pair is monitored via the multiplexer. Once a reading for all three muscular groups is obtained, the performed gesture can be determined by its unique activation. This is encoded to an integer value and transmitted to the PC where the gesture can be translated into a mouse movement on the screen.

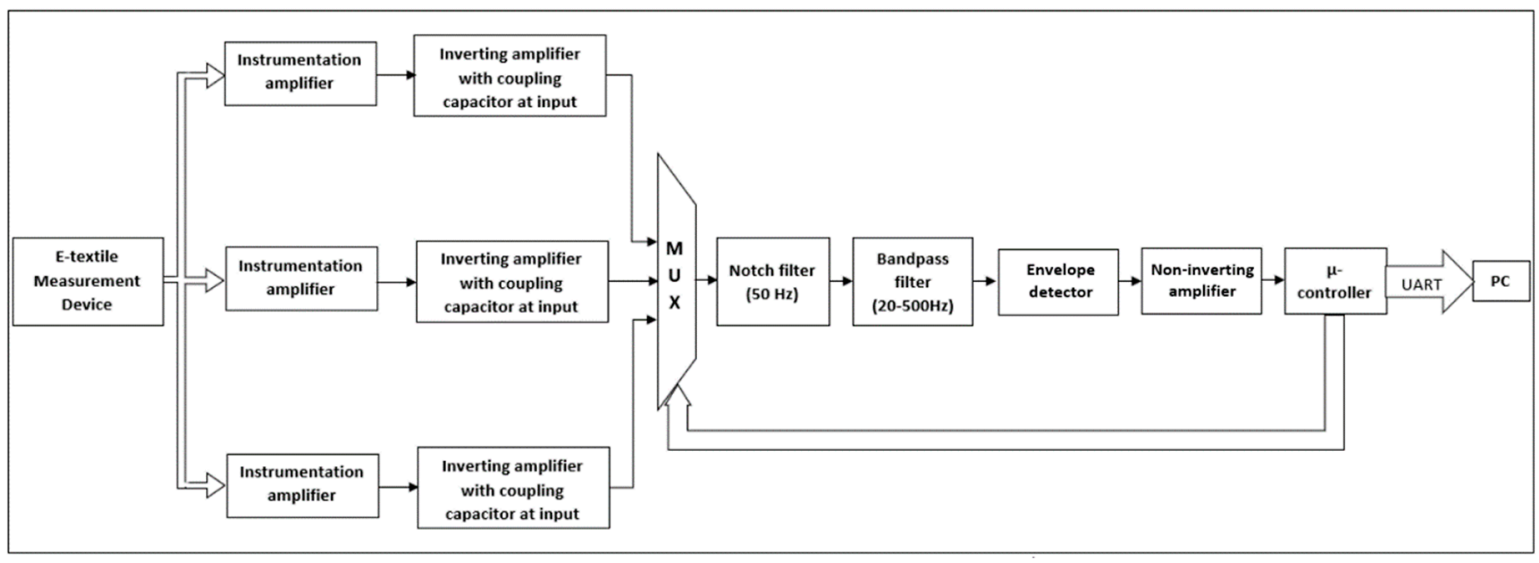

Figure 1. Final full system block diagram for the e-textile gesture controller via EMG monitoring.

\section{Fabrication of Screen-Printed EMG Electrodes on Textiles}

Figures 2 and 3 show the printed electrodes on fabric; produced using a screen-printing process by depositing 4 -functional layers in turn, on a polyester cotton fabric. Firstly, an "Interface layer" (Smart Fabric Inks Ltd., Southampton, UK-Fabinks UV-IF1004) is printed which smooths the surface of the fabric to improve printability for the subsequent conductive layer. This conductive layer (Fabinks TC-C4007) is a silver polymer ink for the conductive tracks. This is then encapsulated using the same ink as the interface layer; insulating the conductive layer from unintended skin contact, only the ends remain open for contact to the skin at one end and connection to the processing circuitry at the other. The final layer is a stencil printed conductive carbon rubber paste (Fabinks TC-E0001) covering one of the exposed conductor areas, providing a dry electrode contact to the skin, the blue area consisting of just the base silicone rubber provides improved adhesion to the fabric.

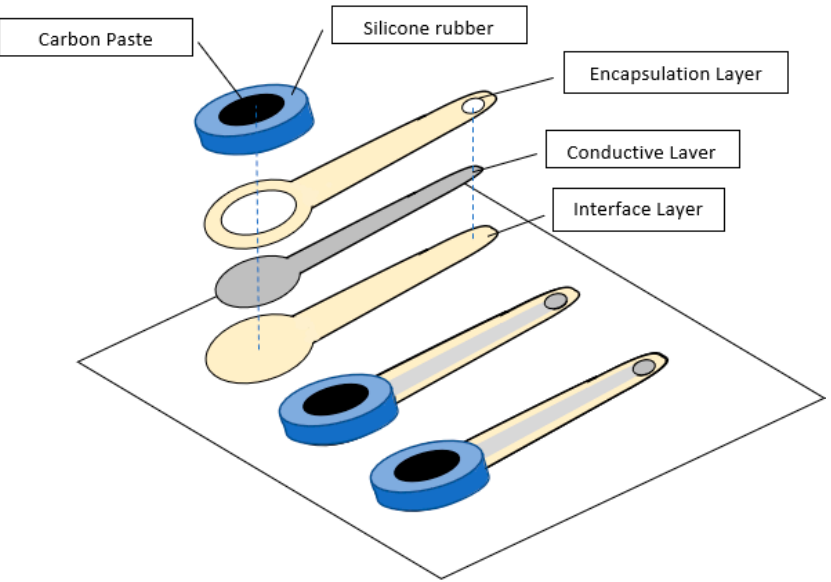

Figure 2. Exploded view of printed electrodes. 


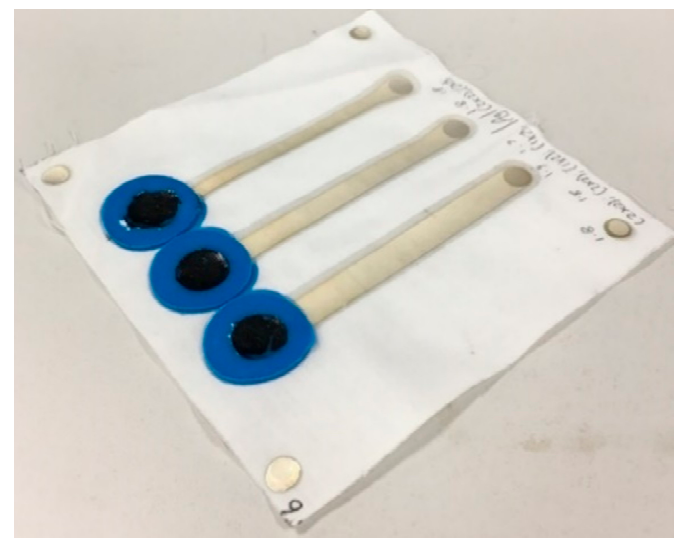

Figure 3. Complete screen-printed electrodes.

The e-textile sensor system in this study is worn on the right forearm, with electrodes placed to target specific muscles. Reviewing the muscular makeup of the forearm, Figure 4, and in particular the superficial muscles, those closest to the skin thus providing an EMG measurement non-invasively with surface electrodes, it was decided to target three muscular groups: 1 . The Brachioradialis, 2. The Extensor group on the outer forearms, 3 . The Flexor group on the inner forearm.
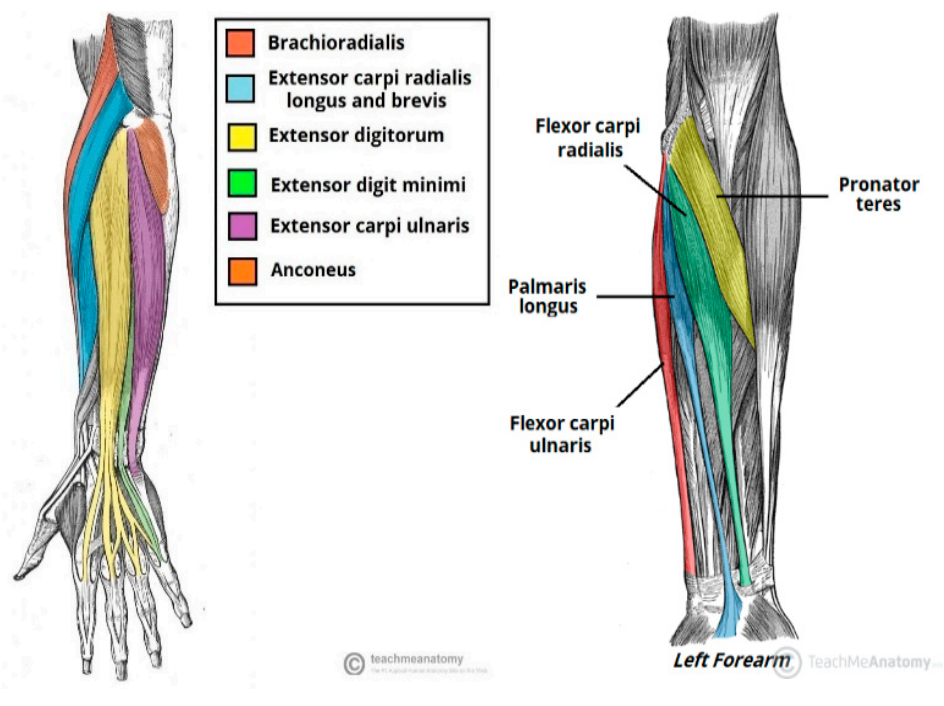

Figure 4. Muscles in the forearm (left) reproduced from [2].

A binary approach was chosen meaning each group is defined as either active of inactive thus providing up to 8 unique activation patterns, i.e. detectable gestures. The final e-textile sensors, Figure 5, uses 3 electrode pairs to take measurements from the 3 muscular groups with an additional reference electrode aligned with the Ulna, a bone running down the back of the forearm. 


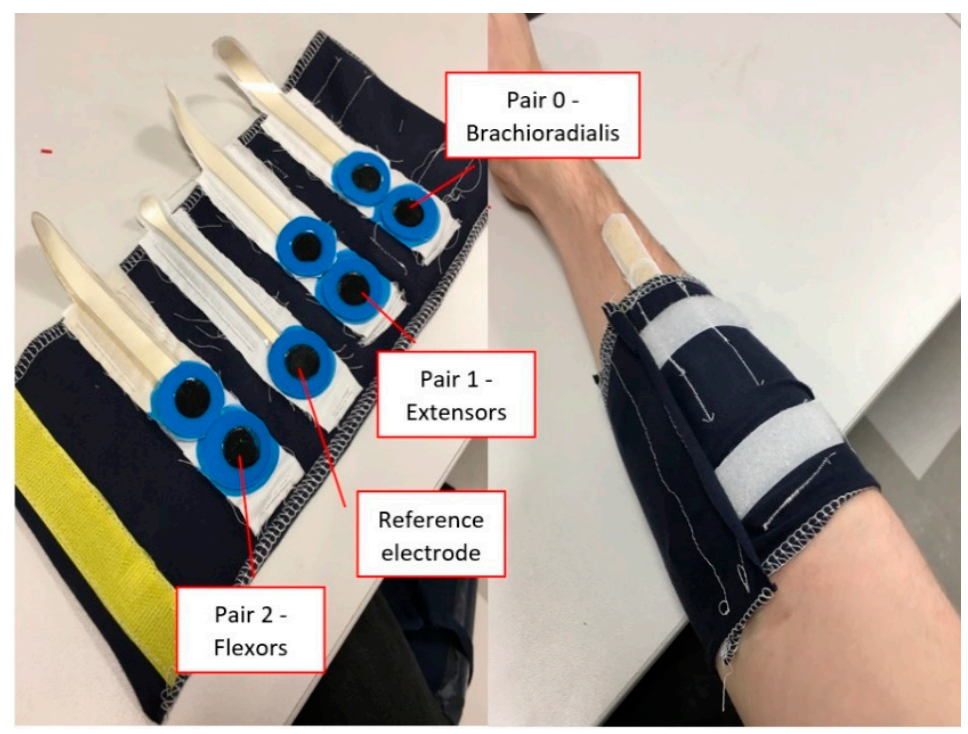

Figure 5. Final measurement device.

\section{E-Textile Sensor System Testing}

The e-textile was tested with different potential gestures [3], allowing activation patterns to be acquired from which to select the final gestures. These were selected based on those which were most comfortable and intuitive for the user to perform without having any overlapping activation patterns between other selected gestures. From a theoretical 8 gestures, it was possible to detect 5 uniquely. The final 5 gestures selected with corresponding activation patterns ("brachioradialis, extensors, flexors") are: hand relaxed"000", wrist flexed-“X01", wrist extended-" $110 "$, fingers spread-“ $010 "$ and fist" 111 ", where " 1 " means an active muscle, " 0 " means inactive and " $X$ " means "don't care". A sixth gesture (pinch) was originally thought to be feasible with an activation pattern "011"; however, the signal magnitude was much lower than the other 5 so its detection proved problematic.

With the gestures and unique activation patterns determined, the microcontroller applies minor error correction before translating the gesture to a cursor movement; the 5 gestures above correspond to: no movement, right, left, downward and upward mouse movements, respectively. System accuracy versus switching time before and after error correction (BEC/AEC) is summarized in Figure 6. It shows error correction improves accuracy by $\sim 1 \%$; however, in many cases an incorrect movement cannot be fully corrected and defaults to no movement. This is still an error, thus not contributing to the percentage improvement; however, it does give significant qualitative improvement in the mouse movement. An overall accuracy of $\sim 93 \%$ is observed for switching delays $\geq 150 \mathrm{~ms}$. Below this, the system accuracy decreases considerably. This is due to the response time of the envelope detector thus higher accuracy at smaller switching times could be achieved with a more sophisticated envelope detector. 


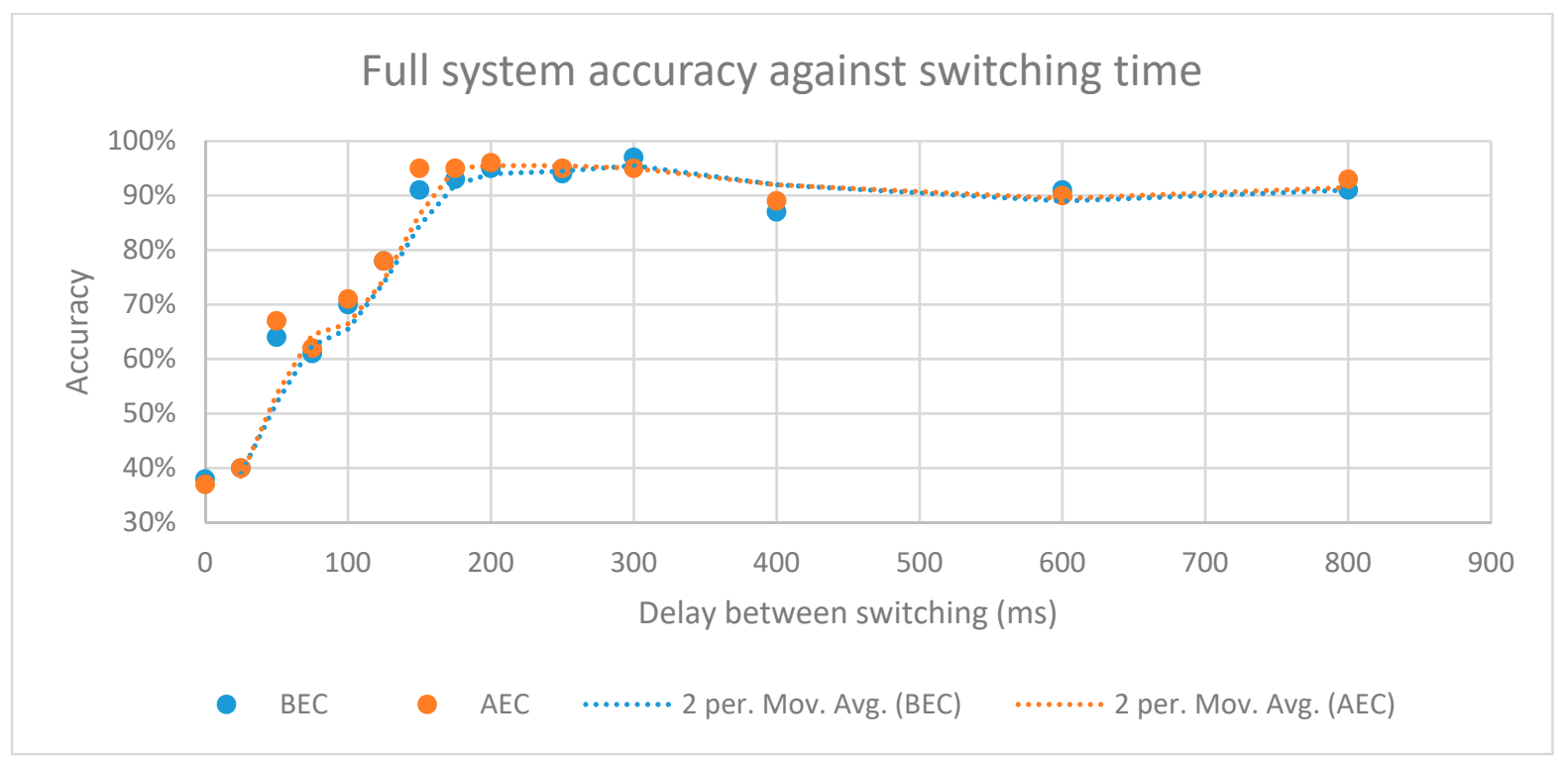

Figure 6. Full e-textile sensor system response against switching time.

\section{Conclusions}

In this paper a complete screen-printed EMG electrode based wearable e-textile measurement system has been designed and tested. The system can detect five unique hand gestures with an overall accuracy of $\sim 93 \%$ when operating with a switching delay of $150 \mathrm{~ms}$ or greater. Were this study to be developed further the next step would be to incorporate the processing circuitry into the e-textile device [4], as opposed to the breadboarded solution currently used. It would also be beneficial to incorporate a Bluetooth connection allowing for the device to be completely self-contained without the user having to be "plugged in". Following this the device could be developed to allow it to control other devices such as a robotic limb as in [5].

Acknowledgments: The authors gratefully acknowledge the financial support from the WEARPLEX EU H2020 project (ID: 825339) - wearplex.soton.ac.uk.

Institutional Review Board Statement: The study was conducted according to the guidelines of the Declaration of Helsinki, and approved by the Ethics Committee of the University of Southampton (ERGO/FPSE/53044-approved 1/11/2019).

Informed Consent Statement: Informed consent was obtained from all subjects involved in the study.

Data Availability Statement: The amplifier circuit diagram and data produced in this study is available to use here: https://doi.org/10.5258/SOTON/D1707.

\section{References}

1. Rawat, S.; Vats, S.; Kumar, P. Evaluating and exploring the MYO ARMBAND. In Proceedings of the 2016 International Conference System Modeling \& Advancement in Research Trends (SMART), Moradabad, India, 25-27 November 2016.

2. Jones. "Muscles of the Upper Limb," Teach Me Anatomy, 15 August 2020. Available online: https://teachmeanatomy.info/upper-limb/muscles/ (accessed on 7 October 2020).

3. Farina, D.; Lorrain, T.; Negro, F.; Jiang, N. High-density EMG E-Textile systems for the control of active prostheses. In Proceedings of the 2010 Annual International Conference of the IEEE Engineering in Medicine and Biology, Buenos Aires, Argentina, 31 August-4 September 2010.

4. Komolafe, A.; Torah, R.; Wei, Y.; Nunes-matos, H.; Li, M.; Hardy, D.; Dias, T.; Tudor, M.; Beeby, S. Integrating flexible filament circuits for e-textile applications. Adv. Mater. Technol. 2019, 4, 1900176.

5. Lopez, J. Myoelctric control. YouTube, 12 September 2016. Available online: https://www.youtube.com/watch?v=0eQf3sWtRj4 (accessed on 17 March 2020). 